\title{
Modeling and optimization of white paint back reflectors for thin-film silicon solar cells
}

\author{
Benjamin Lipovšek, ${ }^{1, a)}$ Janez Krč, ${ }^{1}$ Olindo Isabella, ${ }^{2}$ Miro Zeman, ${ }^{2}$ and Marko Topič ${ }^{1}$ \\ ${ }^{1}$ Faculty of Electrical Engineering, University of Ljubljana, Tržaška 25, 1000 Ljubljana, Slovenia \\ ${ }^{2}$ Electrical Energy Conversion Unit/DIMES, Delft University of Technology, P.O. Box 5053, 2600 GB Delft, \\ The Netherlands
}

(Received 3 August 2010; accepted 6 October 2010; published online 29 November 2010)

\begin{abstract}
Diffusive dielectric materials such as white paint have been demonstrated as effective back reflectors in the photovoltaic technology. In this work, a one-dimensional (1D) optical modeling approach for simulation of white paint films is developed and implemented in a 1D optical simulator for thin-film solar cells. The parameters of white paint, such as the paint film thickness, the pigment volume concentration (PVC), and the pigment/binder refractive index ratio (RIR), are examined and optimized to achieve the required optical properties for back reflector application. The simulation trends indicate that white paint back reflectors with sufficient film thickness and higher PVC and RIR values exhibit improved reflectivity characteristics which results in an increased long-wavelength quantum efficiency of thin-film silicon solar cells. The simulation results based on the 1D model agree very well with the experimental data obtained from reflectance measurements of various white paint compositions and quantum efficiency measurements of amorphous silicon solar cells with white paint back reflectors. (C) 2010 American Institute of Physics.
\end{abstract}

[doi:10.1063/1.3512907]

\section{INTRODUCTION}

In thin-film silicon solar cells, advanced concepts of light trapping need to be employed to boost the conversion efficiency of the cells. ${ }^{1}$ To lengthen the optical paths and thus increase the absorption in thin absorber layers, light scattering at textured interfaces in combination with highly reflective back contacts and anti-reflective coatings at front is utilized in state-of-the-art solar cells. ${ }^{2}$ With respect to light trapping, the back contact (reflector) plays an important role in providing high reflection of the long-wavelength light that is not efficiently absorbed during the first passing through the thin absorber layer. Efficient light scattering at the back reflector is also highly desired, leading to additional prolongation of optical path lengths. Back reflectors in thin-film silicon solar cells are mainly realized by a silver (Ag) or aluminum (Al) film in combination with a thin $\mathrm{ZnO}$ transparent conductive oxide (TCO) layer, which assures better reflectivity and hence lower optical losses in the metal layer. ${ }^{3,4}$ These reflectors (especially Ag-based) exhibit excellent electrical and relatively good optical properties. However, in the case of texturing they suffer from the effect of surface plasmon absorption which can noticeably reduce their reflectivity characteristics. ${ }^{5}$ Furthermore, there are also other drawbacks related to metal back reflectors, such as relatively high production costs and, if not perfectly sealed, the sensitivity of the contact material to the moisture in the environment. ${ }^{6}$ Therefore, alternative back reflector concepts such as back reflectors based on diffusive dielectric materials are being investigated. ${ }^{6}$ Among them, white paint in combination with a TCO back contact presents one of the promising solutions. ${ }^{6,7}$ In this concept, the white paint should act as

${ }^{a)}$ Electronic mail: benjamin.lipovsek@fe.uni-lj.si. a good reflector and efficient diffuser at the same time, whereas the TCO layer [either $\mathrm{ZnO}$ :B deposited by lowpressure chemical vapor deposition (LP-CVD) (Ref. 8), or $\mathrm{ZnO}$ :Al deposited by physical vapor deposition (PVD) (Ref. 9)] should provide a good electrical contact for the cell. Inexpensive commercially available white paints that could be deposited by means of simple printing or spraying techniques are being considered for industrial applications. ${ }^{6}$

To simulate white paints in the role of back reflectors in thin-film solar cells and to optimize their optical properties, an appropriate optical model is required. Different modeling approaches of white paint's optical properties have already been reported, such as the radiative transfer models (e.g., the N-flux method ${ }^{10-12}$ and the Monte Carlo method ${ }^{13}$ ). However, the applicability of these models in simulations of complete thin-film solar cells with multilayer structures in which coherent effects of nonscattered light (at the front side of the cell) as well as incoherent propagation of scattered light (at randomly textured interfaces and in white paint films) has not yet been demonstrated.

In this paper we present a one-dimensional (1D) optical modeling approach which enables simulation of the external reflectivity properties of white paint back reflectors and which is fully applicable to 1D simulations of the entire thinfilm solar cells with the existing optical simulators. ${ }^{14-16}$ The structural characteristics of white paint, such as the thickness of the paint film $(d)$, the pigment volume concentration (PVC), the refractive index ratio (RIR) between the pigment and binder materials, and the pigment particle size, are considered as the input parameters in these simulations. First, the model is integrated into the semicoherent optical simulator SUNSHINE, which has been widely used for simulation of thin-film solar cells with flat and textured interfaces, ${ }^{16}$ and 


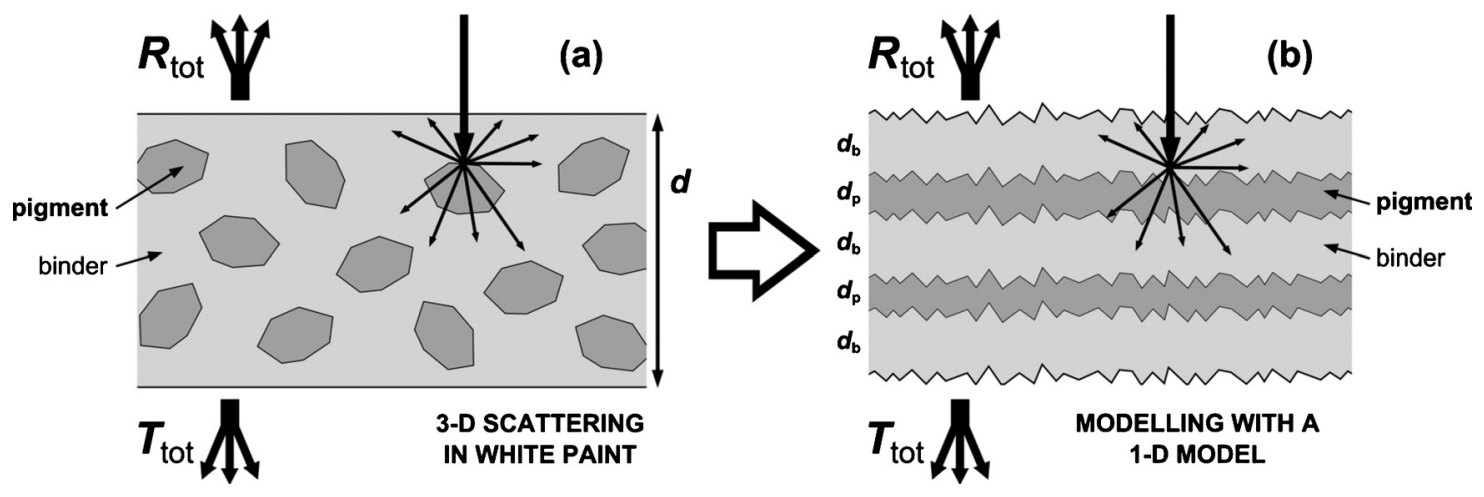

FIG. 1. Three-dimensional light scattering in actual white paint (a) and the proposed 1D optical modeling approach (b).

validated with experimental data. Then, simulations are employed to investigate and optimize the reflectivity properties of different white paint films. The effects of $d$, PVC and RIR on the total reflectance $\left(R_{\mathrm{tot}}\right)$ of the films are shown. Finally, simulations of thin-film amorphous silicon $(a-\mathrm{Si}: \mathrm{H})$ solar cells with different white paint back reflectors are carried out. The improvements in the long-wavelength external quantum efficiency (EQE) of the solar cells deposited on flat and textured superstrates are indicated with respect to the optical optimization of white paints.

\section{WHITE PAINT MODELING}

Basically, the structure of a dry paint film can be represented by a mixture of two primary components: the pigment and the binder. The pigment defines the color of the paint, whereas the binder is used as the matrix in which the pigment particles are dispersed, forming a film [Fig. 1(a)]. Additionally, other components like volatile substances (carriers) and additives are included in the paint composition to improve its viscosity, drying, and stability characteristics. ${ }^{17}$ The external optical properties of dry paint films, however, can mainly be assigned to the pigment and the binder. Therefore, only these two were taken into account in our optical analysis. As the pigment in the commercially available white paints, rutile titanium dioxide $\left(\mathrm{TiO}_{2}\right)$ particles with the refractive index $n_{\mathrm{p}}=2.74(\lambda=550 \mathrm{~nm})$ and typical particle diameter of $300 \mathrm{~nm}$ are most commonly employed, ${ }^{12}$ whereas various polymeric latex-based materials with typical refractive indices $n_{\mathrm{b}}=1.4-1.7$ are used as the binder. ${ }^{18}$ In our simulations, the values of the wavelength-dependent refractive index and the absorption coefficient of rutile $\mathrm{TiO}_{2}$ were taken from literature (average of the values for ordinary and extraordinary rays), ${ }^{19,20}$ whereas the refractive index and the absorption coefficient of the polymeric binder used were determined experimentally, employing the reflectancetransmittance method. ${ }^{21}$

Since the paint medium (i.e., the binder with the dispersed pigment particles) is assumed to be isotropic, it is possible to simulate the optical mechanisms taking place within the paint film (propagation of light through the pigment and binder materials; reflection, transmission and scattering at the pigment interfaces) by means of a 1D model. In this work, we develop a 1D optical model in which the actual structure of a white paint film [Fig. 1(a)] is represented by a virtual multilayer stack consisting of two interchanging layers representing the pigment and the binder materials [Fig. 1(b)]. The thickness of the entire stack is equal to the thickness of the paint film $(d)$, whereas the thickness of each pigment layer $\left(d_{\mathrm{p}}\right)$ is equal to the average path length of light traveling through a pigment particle $(\sim 200 \mathrm{~nm}$ for a typical spherical $\mathrm{TiO}_{2}$ particle with a diameter of about $300 \mathrm{~nm}$ ). The thicknesses of the binder layers and the number of all the layers in the stack are determined to match the PVC at certain $d$ of the actual white paint film.

Multiple reflections and transmissions of light at the surfaces of the pigment particles are translated to reflections and transmissions at the interfaces between the layers in the 1D model. The total reflectance $\left(R_{\mathrm{tot}}\right)$ and the total transmittance $\left(T_{\text {tot }}\right)$ at a pigment/binder interface (including specular and diffused light components) are determined based on the wavelength-dependent complex refractive indices of the pigment $\left(N_{p}\right)$ and the binder $\left(N_{b}\right)$ according to Eqs. (1) and (2) (Ref. 22)

$$
\begin{aligned}
& R_{\mathrm{tot}}=\left|\frac{N_{p}-N_{b}}{N_{p}+N_{b}}\right|^{2}, \\
& T_{\text {tot }}=1-R .
\end{aligned}
$$

The scattering (diffusive) properties of the pigment particles, both in reflection and transmission, are in the model applied to the interfaces between the layers. For this reason, a certain degree of surface nanotexture is introduced at each interface. To define the level of scattering at the interfaces, it was found out that the equations from the scalar scattering theory, ${ }^{23,24}$ which are commonly employed to determine the level of scattered light at randomly nanotextured interfaces in thin-film solar cells, ${ }^{14,25,26}$ render a sufficient approximation of the actual scattering of white paint films. Thus, to calculate the wavelength-dependent haze parameter (ratio of the diffused to total light) for the reflected $\left(H_{\mathrm{R}}\right)$ and the transmitted light $\left(H_{\mathrm{T}}\right)$ at an interface in the $1 \mathrm{D}$ model, the following equations are utilized:

$$
H_{\mathrm{R}}=1-\exp \left[-\left(\frac{4 \pi \times \sigma_{\mathrm{rms}} \times n_{\mathrm{inc}}}{\lambda}\right)^{2}\right],
$$




$$
H_{\mathrm{T}}=1-\exp \left[-\left(\frac{2 \pi \times \sigma_{\mathrm{rms}} \times\left|n_{\mathrm{inc}}-n_{\mathrm{out}}\right|}{\lambda}\right)^{3}\right] .
$$

In the above equations, the wavelength of light in vacuum is indicated by $\lambda$, whereas $n_{\text {inc }}$ and $n_{\text {out }}$ represent the refractive indices of the incident layer and the layer in transmission, respectively. In our case, they are assigned to the pigment and binder materials, depending on the interface and the direction of light propagation (forward or backward). The only parameter in the 1D model used to describe the interface texturisation is $\sigma_{\text {rms }}$, which represents the root-mean-square roughness of the surface nanotexture applied to all the interfaces in the white paint multilayer system. The $\sigma_{\text {rms }}$ parameter in this case can mostly be related to the size of the pigment particles. However, since its direct relation to the average pigment particle diameter has not been derived so far, it is taken as the fitting parameter which can be extracted from experimental data. Assuming the above mentioned relation, the same $\sigma_{\mathrm{rms}}$ is considered for white paint compositions having the same average pigment particle diameter. In our simulations where the average pigment particle diameter was $300 \mathrm{~nm}, \sigma_{\text {rms }}=50 \mathrm{~nm}$ was found to be the best fitting solution, rendering good agreement between the simulations and measurements of white paint compositions with different $d$ and PVC values. Simulations revealed that changing $\sigma_{\text {rms }}$ does not only affect the level of scattering, but also affects the slope of the long-wavelength $R_{\text {tot }}$ curve of the white paint film. Furthermore, as the angular distribution function (ADF) of the scattered light at the textured interfaces (pigment particles) in the 1D model, the Lambertian (cosine) distribution function was employed both in reflection and transmission, as determined by the angular resolved scattering (ARS) measurements of paint film samples (see further).

The developed 1D model of white paint films, in which the multiple reflection, transmission, scattering, and propagation of light rays are all taken into account, was integrated into the semicoherent optical simulator SUNSHINE. In the semicoherent analysis, the direct nonscattered light is assumed to be coherent inside the thin layers and can thus be treated in terms of electromagnetic waves, whereas propagation of the incoherent scattered component is evaluated by the method of ray-tracing projected to one dimension. In white paint, most of the light becomes incoherent due to the multiple-scattering process. In the following we show that using the SUNSHINE simulator upgraded with the presented 1D model, the external optical properties of white paint films of different composition with respect to $d$, PVC, and RIR can be determined. Additionally, by adding the solar cell layers on top of the white paint multilayer stack, the entire solar cell structures with white paint back reflectors can also be simulated, and thus the performance of thin-film solar cells with different white paint back reflectors can be studied.

\section{EXPERIMENTAL PROCEDURES}

White paint samples required for verification of the model were prepared by mixing the rutile $\mathrm{TiO}_{2}$ pigment with a mean diameter of $300 \mathrm{~nm}$ and a polymeric binder in a mortar grinder. The PVC, which is defined as the ratio of the pigment volume to the entire paint volume, was varied from
$5 \%-30 \%$. The prepared paints were applied on borosilicate glass substrate using the "doctor blading" technique ${ }^{27}$ and dried out at room temperature $\left(\sim 20{ }^{\circ} \mathrm{C},>3 \mathrm{~h}\right)$. The paint film thickness $(d)$ of the samples spanned from 14-150 $\mu \mathrm{m}$. Additionally, a $100 \mu \mathrm{m}$ thick sample of the commercially available white paint Tipp-Ex was also prepared as a reference.

The total and diffused reflectance $\left(R_{\text {tot }}, R_{\text {dif }}\right)$ of all the back reflector samples were determined experimentally in the wavelength range of $300-1500 \mathrm{~nm}$ by means of the PerkinElmer Lambda 950 spectrophotometer equipped with an integrating sphere. The ADF of the reflected light was measured using an ARS system. ${ }^{25}$ All the measurements were performed in air with the illumination incident on the sample film side. This way we avoided the effects of (total) back reflections at the glass/air surface which can occur for light scattered in large angles if the samples were illuminated from the glass side. However, in simulations of complete solar cells with white paint back reflectors, all these reflections at the interfaces between the layers located above the white paint back reflector were taken into the account.

Thin-film $a$-Si:H $p-i-n$ solar cells were deposited in a plasma-enhanced chemical vapor deposition reactor on two types of superstrates: (i) glass/ZnO:Al with a flat surface and (ii) glass $/ \mathrm{SnO}_{2}: \mathrm{F}$ (Asahi U) with a textured surface (natural texture with $\sigma_{\text {rms }} \approx 40 \mathrm{~nm}$ ). Both front TCO films were about $800 \mathrm{~nm}$ thick. The thickness of the intrinsic $i-a-\mathrm{Si}: \mathrm{H}$ absorber layer was $300 \mathrm{~nm}$. To form the back electrical contact of the cell with white paint back reflectors, a $350 \mathrm{~nm}$ thick layer of $\mathrm{ZnO}: \mathrm{Al}$ was magnetron-sputtered at lowtemperature $(300 \mathrm{~K})$ on top of the $n-a-\mathrm{Si}: \mathrm{H}$ layer at a deposition rate of $3.5 \mathrm{~nm} / \mathrm{min}$. On top of the $\mathrm{ZnO}$ :Al layer, white paint films were applied as the back reflectors. The active area of the fabricated $a-\mathrm{Si}: \mathrm{H}$ cells was $0.6 \mathrm{~cm}^{2}$. To demonstrate the improvements related to the optimization of white paint back reflectors, EQEs of the fabricated $a-\mathrm{Si}: \mathrm{H}$ solar cells were measured. The short-circuit current densities $\left(J_{\mathrm{SC}}\right)$ of the cells were calculated from the measured EQE curves by considering the AM 1.5 spectrum.

\section{RESULTS AND DISCUSSION}

\section{A. Results of optical characterization}

The reflectivity and scattering properties of white paint films, which are crucial for efficient back reflectors in thinfilm solar cells, are characterized by the total reflectance $\left(R_{\text {tot }}\right)$, the haze parameter for the reflected light $\left(H_{\mathrm{R}}\right)$, and the ADF of the reflected scattered light. In Fig. 2, the measured $R_{\text {tot }}$ and $H_{\mathrm{R}}$ of a commercial white paint Tipp-Ex $(100 \mu \mathrm{m}$ thick film on a glass substrate) are compared with the measurements of a textured Ag reflector (100 nm thick Ag film on $\mathrm{SnO}_{2}: \mathrm{F}$ Asahi $\mathrm{U}$ substrate with $\sigma_{\text {rms }} \approx 40 \mathrm{~nm}$ ). The results show that $R_{\text {tot }}$ of the textured Ag reflector is generally higher than $R_{\text {tot }}$ of the white paint sample, which peaks at $\lambda=440 \mathrm{~nm}\left(R_{\mathrm{tot}}=0.85\right)$ and then gradually decreases toward longer wavelengths. The abrupt drop of white paint's $R_{\text {tot }}$ in the low-wavelength region $(\lambda<420 \mathrm{~nm})$ is related to the absorption in $\mathrm{TiO}_{2}$ particles [indirect allowed transitions in $\mathrm{TiO}_{2}$ at about $3.02 \mathrm{eV}$ (Ref. 11)] and is not critical from the 


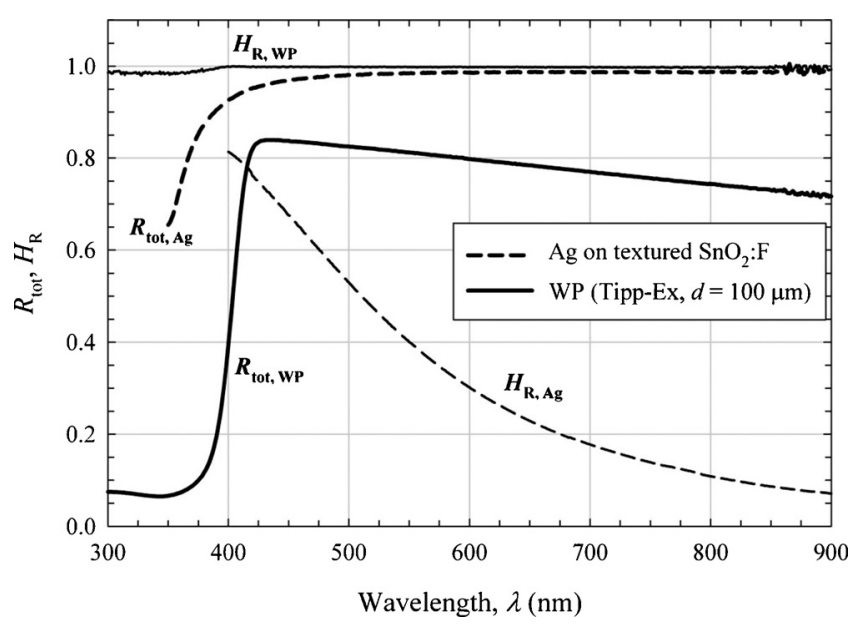

FIG. 2. The measured $R_{\text {tot }}$ and $H_{\mathrm{R}}$ of the white paint sample compared to the textured $\mathrm{Ag}$ back reflector.

back reflector point of view, since the short-wavelength light $(\lambda<550 \mathrm{~nm})$ is efficiently absorbed in the absorber layers of the solar cell before reaching the back reflector. The $H_{\mathrm{R}}$ measurement results in Fig. 2 show that the white paint sample exhibits extremely high, almost ideal $H_{\mathrm{R}} \approx 1$ throughout the wavelength range, whereas $H_{\mathrm{R}}$ of the textured Ag reflector follows exponential decay.

The measured ADFs of the reflected scattered light for the white paint sample and the textured Ag-based back reflector are shown in Fig. 3 (measured in a plane perpendicular to the sample). The results show that compared to the Ag reflector, the white paint sample exhibits much broader ADF which approaches to the Lambertian (cosine) distribution (also plotted in Fig. 3). Similar broad ADF curves were obtained also for other white paint samples of different film thickness and composition (not shown here). These results demonstrate excellent scattering capabilities of white paint (high $H_{\mathrm{R}}$, broad ADF), which are far superior to those of conventional metal-based reflectors. However, relatively lower $R_{\text {tot }}$ values call for further optimization of white paint

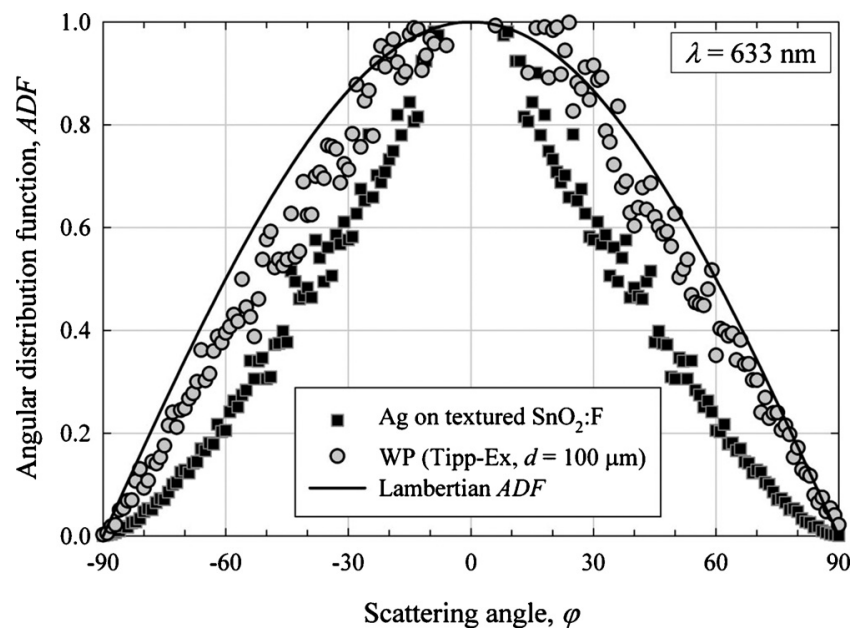

FIG. 3. The measured ADF $(\lambda=633 \mathrm{~nm})$ of the white paint sample compared to the textured Ag back reflector. The Lambertian (cosine) ADF is also plotted (full line).

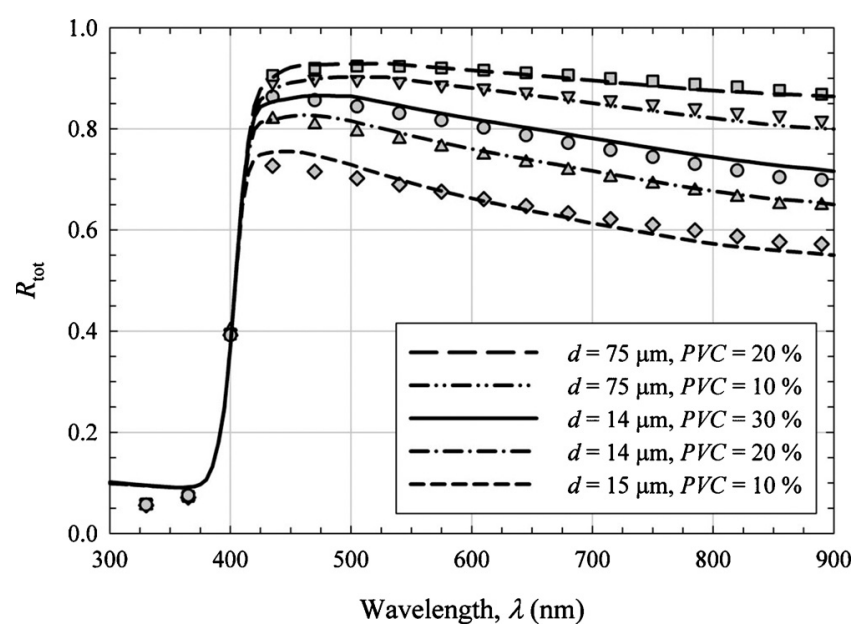

FIG. 4. The simulated (lines) and the measured (symbols) total reflectance of a number of white paint samples exhibiting different $d / \mathrm{PVC}$ combinations.

film composition and thickness. For this purpose, the developed 1D optical model can be efficiently applied.

\section{B. Verification of the 1D optical modeling approach}

To verify the proposed $1 \mathrm{D}$ modeling approach, we simulated the total reflectance of different white paint films and compared the results with the measurements. The input parameters for the simulations were determined according to the properties of the white paint films (pigment and binder materials, paint film thickness, PVC), as described in Sec. II. The $\sigma_{\text {rms }}$ parameter which presents the only fitting parameter was set to $50 \mathrm{~nm}$ in all the simulations.

The measured (symbols) and the simulated (lines) $R_{\text {tot }}$ curves of the selected white paint samples are presented in Fig. 4. The simulated results are in good agreement with the measurements for all the samples with different $d$ and PVC. A trend of increased $R_{\text {tot }}$ as a result of increased $d$ and PVC can be observed (discussed later), whereas for the films with smaller $R_{\text {tot }}$, higher transmission losses can be detected. The $H_{\mathrm{R}}$ parameter, which was calculated from the simulated diffuse reflectance divided by the total reflectance, is close to unity for all the white paint films, similar to $H_{\mathrm{R}}$ of the white paint Tipp-Ex shown in Fig. 2.

\section{Simulation and optimization of the optical properties of white paint films}

The level of $R_{\text {tot }}$ of a white paint film, as indicated by Fig. 4, is closely related to the combination of the film thickness $(d)$ and the PVC. By means of the verified 1D modeling approach, the role of each of the two parameters can be investigated independently and optimized with respect to higher $R_{\text {tot }}$. In these simulations, the pigment and binder materials remain unchanged, and thus RIR is kept constant at $1.83(\lambda=650 \mathrm{~nm})$. The optimization results will be shown for the selected wavelength $\lambda=650 \mathrm{~nm}$, representing the long-wavelength region which is of interest in $a$-Si:H solar cells from the point of view of high back-reflection. However, since simulations indicate that increasing $R_{\text {tot }}$ at $\lambda$ $=650 \mathrm{~nm}$ results in an increased $R_{\text {tot }}$ at longer wavelengths 


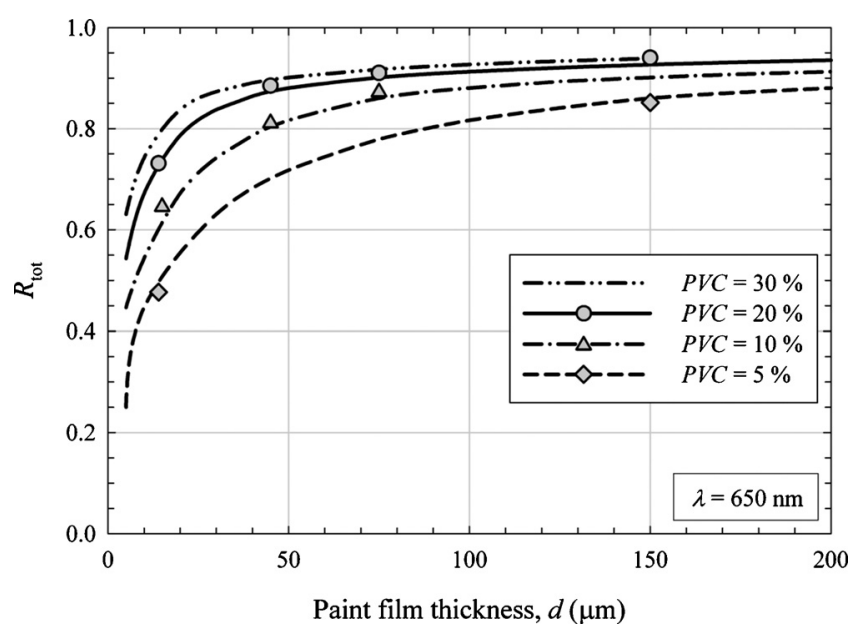

FIG. 5. The simulated (lines) and the measured (symbols) total reflectance of white paints with different PVC $\mathrm{s}$ as a function of film thickness.

as well, the analysis is also valid for microcrystalline silicon solar cells where longer wavelengths (e.g., $\lambda>800 \mathrm{~nm}$ ) should be considered.

In Fig. 5, the simulated $R_{\text {tot }}$ of white paint films is shown as a function of film thickness for four different PVC's. The simulations are again in good agreement with measured data, confirming the optimization trends obtained by the 1D modeling. All four plots in Fig. 5 indicate similar behavior of $R_{\text {tot }}$, which increases dramatically as $d$ increases from 10 to $100 \mu \mathrm{m}$. This can be explained by the fact that the thicker the film is, the more pigment/binder interfaces there are in the coating (in the actual film as well as in the 1D representation). Subsequently, with the increased number of interfaces, the cumulative $R_{\text {tot }}$ is also increased since the light can be reflected back more times. However, due to the light absorption in the paint film (both the pigment and especially the binder are absorptive in the long-wavelength region), a saturation of $R_{\text {tot }}$ below the ideal value $R_{\text {tot }}=1$ occurs at certain film thickness. Modeling can be used to determine this saturation region for the paints with different PVC's, and thus evaluate the minimal required thickness of the film that still renders the highest $R_{\text {tot }}$. Simulations indicated that if the absorption losses in the white paint film were smaller, the optimal thickness would shift toward larger values, and higher maximal $R_{\text {tot }}$ would also be achieved (effect not shown here).

In Fig. 6 the simulated $R_{\text {tot }}$ of white paint films is shown as a function of PVC for different film thicknesses. This way the role and the saturation level of PVC are more clearly presented than in Fig. 5, where PVC was only a parameter. As the PVC increases from $5 \%$ to $30 \%, R_{\text {tot }}$ of the paint film is increased substantially. This can be attributed to the fact that as the PVC is increased, the number of the pigment/ binder interfaces in the same paint film volume is increased as well (also in the 1D representation), leading to higher cumulative $R_{\text {tot }}$. The simulations reveal that saturation of $R_{\text {tot }}$ appears at PVC between 30\%-50\%, indicating the optimal PVC for a certain paint film thickness. It should be noted, however, that the phenomenon of "crowding" (agglomeration) of the pigment, which can start to take place in the paint films at higher PVC values $(>50 \%)$, was not included in our

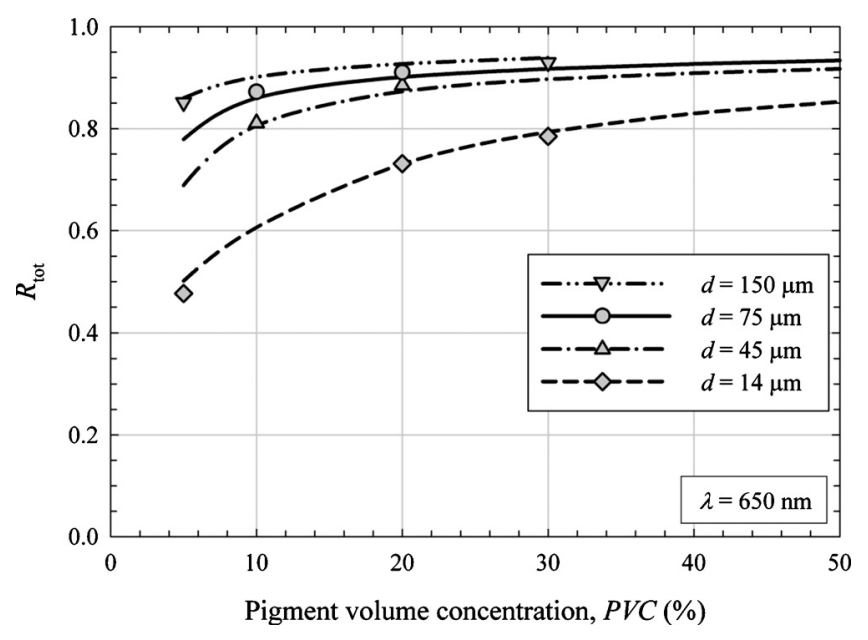

FIG. 6. The simulated (lines) and the measured (symbols) total reflectance of white paints with different film thicknesses as a function of PVC.

simulations. Such agglomeration leads to a reduction in the (effective) pigment/binder interfaces and consequently to a decrease in $R_{\text {tot }}$. Furthermore, structural stability issues may also arise in films with high PVC. ${ }^{17}$

In all the preceding simulations, the pigment/binder RIR of the paint was kept constant at RIR $=1.83\left(n_{\mathrm{p}}=2.69, n_{\mathrm{b}}\right.$ $=1.47$ at $\lambda=650 \mathrm{~nm}$ ). However, since this ratio dictates the total reflectance at a single pigment/binder interface according to Eq. (5) [derived from Eq. (1) by considering only the real parts of the refractive indices in the long-wavelength region), it also presents one of the influencing parameters that affect $R_{\text {tot }}$ of the paint film (besides $d$ and PVC).

$$
R_{\mathrm{tot}} \approx\left(\frac{n_{\mathrm{p}}-n_{\mathrm{b}}}{n_{\mathrm{p}}+n_{\mathrm{b}}}\right)^{2}=\left(\frac{n_{\mathrm{p}} / n_{\mathrm{b}}-1}{n_{\mathrm{p}} / n_{\mathrm{b}}+1}\right)^{2}=\left(\frac{\mathrm{RIR}-1}{\mathrm{RIR}+1}\right)^{2} .
$$

In our simulations, RIR was varied from 1.0 to 2.6 by changing the refractive index of the pigment material $\left(n_{\mathrm{b}}\right.$ was kept constant at 1.47). In Fig. 7, the results at the selected wavelength $\lambda=650 \mathrm{~nm}$ are presented for the case of a white paint film with $d=50 \mu \mathrm{m}$ and $\mathrm{PVC}=20 \%$. Besides the simulated

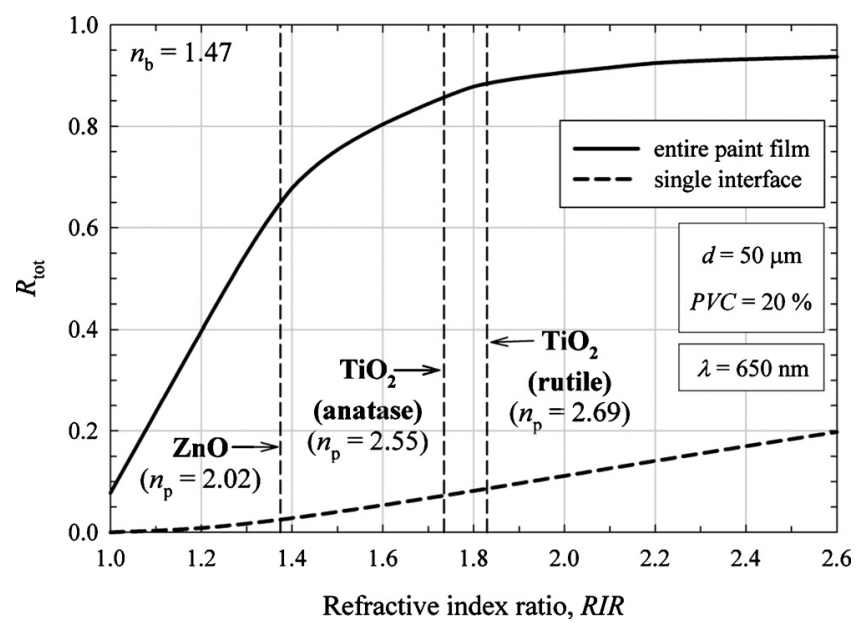

FIG. 7. The simulated total reflectance of a $50 \mu \mathrm{m}$ thick white paint film with $\mathrm{PVC}=20 \%$ as a function of RIR. Also plotted is the reflectance at a single rutile $\mathrm{TiO}_{2}$ pigment/binder interface as a function of RIR (dashed line). The RIR's in the case of three typical white pigments are indicated with vertical lines (the same binder with $n_{\mathrm{b}}=1.47$ is assumed). 

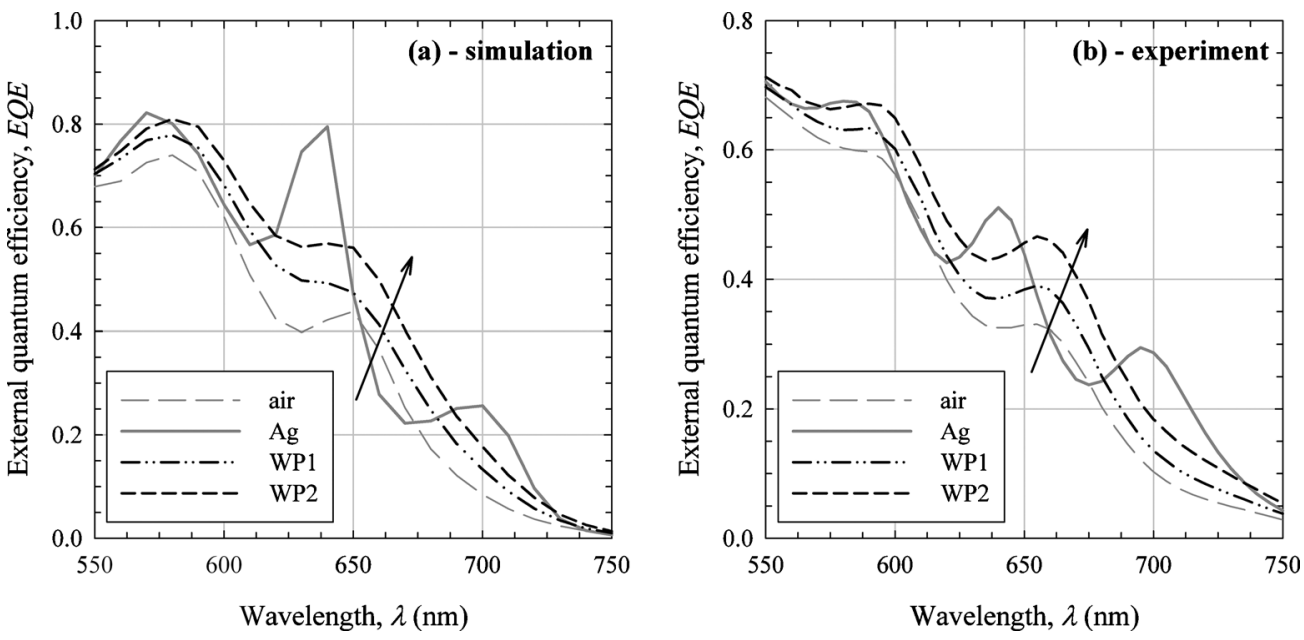

FIG. 8. Simulated (a) and measured (b) EQEs of thin-film $a$-Si:H solar cells on flat ZnO:Al superstrates with different back reflectors.

$R_{\text {tot }}$ of the paint film, $R_{\text {tot }}$ corresponding to the reflectance of a single binder/pigment interface [calculated from Eq. (5)] is also shown. Simulations indicate that RIR plays a crucial role in affecting $R_{\mathrm{tot}}$. The $R_{\mathrm{tot}}$ of the white paint noticeably increases with the RIR and reaches saturation at high RIR values $(>2.2)$. Here, the increase in $R_{\mathrm{tot}}$ is no longer related to the number of pigment/binder interfaces, but instead to the reflectance at a single interface, which increases with increasing RIR. In Fig. 7, the RIRs in the case of three common white pigment materials-rutile $\mathrm{TiO}_{2}$, anatase $\mathrm{TiO}_{2}$, and $\mathrm{ZnO}$ (the corresponding refractive indices at $650 \mathrm{~nm}$ are $2.69,2.55$, and 2.02, respectively) - are indicated by vertical lines. It is evident that among these three, the rutile $\mathrm{TiO}_{2}$-based pigment presents the best choice for achieving high $R_{\text {tot }}$, whereas $\mathrm{ZnO}$ is the least favorable due to its lowest refractive index. Alternatively, instead of changing the pigment material, binders with lower $n_{\mathrm{b}}$ could also be employed in order to increase RIR and thus further increase the total reflectance of the white paint film.

\section{Thin-film solar cells with white paint back reflectors}

By means of the presented 1D model of white paint integrated in the SUNSHINE optical simulator, the EQEs of thin-

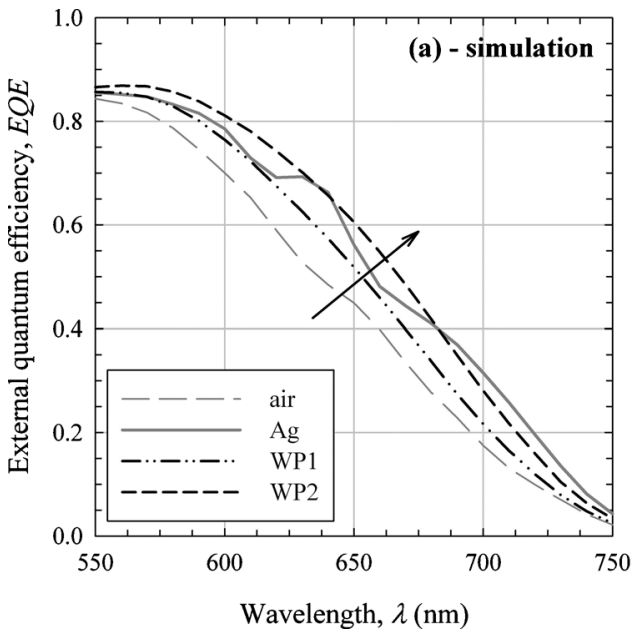

film $a$-Si:H solar cells with different white paint back reflectors were simulated. The solar cells were also fabricated for verification purposes. In the simulations, realistic layer thicknesses of the solar cells (as described previously) and optical properties of $p-a-\mathrm{SiC}: \mathrm{H}, i-a-\mathrm{Si}: \mathrm{H}, n-a-\mathrm{Si}: \mathrm{H}, \mathrm{TCO}(\mathrm{ZnO}: \mathrm{Al}$ and $\mathrm{SnO}_{2}: \mathrm{F}$ ), and $\mathrm{Ag}$ layers were considered. ${ }^{14}$ In the case of textured $\mathrm{SnO}_{2}: \mathrm{F}$ superstrate, the scattering characteristics of the textured $\mathrm{SnO}_{2}: \mathrm{F}$ surface and other nanorough interfaces in the solar cell structure were taken into account. ${ }^{25}$ As the back contact, a $350 \mathrm{~nm}$ thick layer of $\mathrm{ZnO}: \mathrm{Al}$ (followed by the white paint back reflector stack or Ag reflector) was employed.

The simulated (a) and the measured (b) long-wavelength $(\lambda>550 \mathrm{~nm}) \mathrm{EQE}$ curves of $a-\mathrm{Si}: \mathrm{H}$ solar cells deposited on flat $\mathrm{ZnO}: \mathrm{Al}$ and textured $\mathrm{SnO}_{2}: \mathrm{F} \mathrm{TCO} /$ glass superstrates are shown in Figs. 8 and 9, respectively. At shorter wavelengths (below $550 \mathrm{~nm}$ ) the optical properties of back reflectors do not affect the EQE, since the short-wavelength light is efficiently absorbed before reaching the back side of the cell. The results are presented for two types of white paint back reflectors, labeled WP1 $(d=14 \mu \mathrm{m}, \mathrm{PVC}=5 \%)$ and WP2 $(d=150 \mu \mathrm{m}, \mathrm{PVC}=30 \%)$, which represent the two extreme cases of optically poor and optically optimized white paint films, respectively. The EQE of the cell without any back

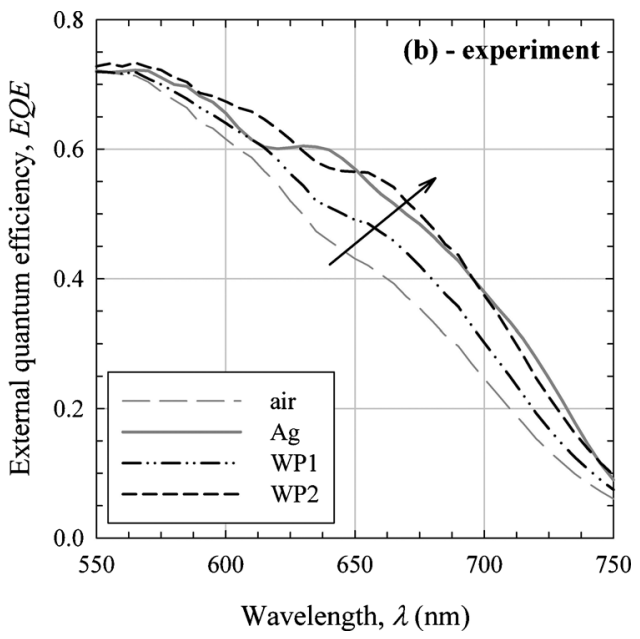

FIG. 9. Simulated (a) and measured (b) EQEs of thin-film $a$-Si: $\mathrm{H}$ solar cells on textured $\mathrm{SnO}_{2}: \mathrm{F}$ superstrates with different back reflectors. 
reflector after the $\mathrm{ZnO}$ :Al layer (air surrounding) and the $\mathrm{EQE}$ of the cell with an $\mathrm{Ag}$ reflector deposited on top of the $\mathrm{ZnO}$ :Al layer are shown for comparison. These results clearly demonstrate the effects of white paint optimization on the performance of the solar cells. As $R_{\mathrm{tot}}$ of the white paint back reflector is increased (by increasing $d$ and PVC of the film), the long-wavelength EQE of the cell is boosted notably for both types of superstrates. Since in the case of cell deposited on flat superstrate the light is scattered only at the white paint reflector, some interference fringes as a consequence of the (coherent) specular light can still be observed in EQE. The measured EQE's confirm the trends observed in the simulated EQE's. However, the measured EQE values are generally lower than the simulated ones (note different $\mathrm{EQE}$ scales), which we attribute mainly to the nonoptimized optical and electrical characteristics of the low-temperature $\mathrm{ZnO}: \mathrm{Al}$ layer as the back contact in the fabricated cells. Nevertheless, the same trends are expected also in electrically optimized solar cells.

The comparison of $J_{\mathrm{SC}}$ values that were calculated from the simulated long-wavelength EQE reveals a relative increase in $10.5 \%$ and $10.7 \%$ if WP2 reflector is employed instead of WP1 in the cell deposited on flat and textured superstrate, respectively. The absolute $J_{\mathrm{SC}}$ values calculated from EQE at $\lambda>550 \mathrm{~nm}$ for the cells with WP2 back reflector are $6.35 \mathrm{~mA} / \mathrm{cm}^{2}$ (flat) and $7.38 \mathrm{~mA} / \mathrm{cm}^{2}$ (textured superstrate). Considering the wavelength spectrum from 350 $750 \mathrm{~nm}$, the corresponding $J_{\mathrm{SC}}$ 's of the cells with WP2 back reflector are $12.25 \mathrm{~mA} / \mathrm{cm}^{2}$ (flat) and $14.66 \mathrm{~mA} / \mathrm{cm}^{2}$ (textured superstrate). These values reach and even exceed the $J_{\mathrm{SC}}$ values obtained with the $\mathrm{Ag}$ back reflector $\left(12.11 \mathrm{~mA} / \mathrm{cm}^{2}\right.$ for the flat and $14.51 \mathrm{~mA} / \mathrm{cm}^{2}$ for the textured superstrate). The same relative trends in $J_{\mathrm{SC}}$ as determined from the simulations are also obtained from the measured EQEs.

\section{CONCLUSIONS}

A 1D optical model was presented that enables simulation of the external optical properties of white paint films as well as entire thin-film solar cells with flat and textured interfaces and white paint back reflectors. In this approach, white paints are modeled as stacks of two interchanging layers corresponding to the pigment and the binder media. Light scattering properties are applied to the interfaces between the layers. The simulation results are in good agreement with the reflectance measurements of a number of different white paint samples and EQE measurements of solar cells with white paint back reflectors. The developed model was employed to investigate the effects of three primary white paint structural parameters - the paint film thickness, the PVC, and the pigment/binder RIR. The simulation results show that generally high values of all three parameters are desired in order to achieve a high total reflectance of the white paint film. White paint films in combination with a conductive $\mathrm{ZnO}: \mathrm{Al}$ layer were then employed as the back reflectors in thin-film $a$-Si:H solar cells deposited on flat and textured superstrates. The characterization results as well as simulations show that optically optimized white paint back reflec- tors can assure good performance of the solar cells on both types of superstrates. We demonstrated that the proposed simple 1D modeling approach presents a useful tool to simulate and optimize white paint back reflectors in photovoltaic devices.

\section{ACKNOWLEDGMENTS}

The work was carried out under subsidy of the Slovenian Research Agency (Project No. J2-0851-1538-08 and program P2-0197) and the Dutch Ministry of Economic Affairs (Project No. EOSLT04029).

${ }^{1}$ A. V. Shah, M. Vaněček, J. Meier, F. Meillaud, J. Guillet, D. Fischer, C. Droz, X. Niquille, S. Faÿ, E. Vallat-Sauvain, V. Terrazzoni-Daudrix, and J. Bailat, J. Non-Cryst. Solids 338-340, 639 (2004).

${ }^{2}$ J. Meier, S. Benagli, J. Bailat, D. Borello, J. Steinhauser, J. Hötzel, L. Castens, J.-B. Orhan, Y. Djeridane, E. Vallat-Sauvain, and U. Kroll, Amorphous and Polycrystalline Thin-Film Silicon Science and Technology, MRS Symposia Proceedings No. 1245 (Materials Research Society, Pittsburgh, 2010), p. 1245-A01-02.

${ }^{3}$ L. R. Dahal, D. Sainju, J. Li, N. J. Podraza, M. N. Sestak, and R. W. Collins, Proceedings of the 34th IEEE Photovoltaic Specialists Conference, Philadelphia, Pennsylvania, 7-12 June 2009, pp. 1702-1707.

${ }^{4}$ J. Hüpkes, T. Wätjen, R. van Aubel, R. Schmitz, W. Reetz, and A. Gordijn, Proceedings of the 23rd European Photovoltaic Solar Energy Conference and Exhibition, Valencia, Spain, 1-5 September 2008, pp. 2419-2421.

${ }^{5}$ J. Springer, A. Poruba, L. Müllerova, and M. Vanecek, J. Appl. Phys. 95, 1427 (2004).

${ }^{6}$ J. Meier, U. Kroll, and A. Büchel, Proceedings of the 31st IEEE Photovoltaic Specialists Conference, Orlando, Florida, 3-7 January 2005, pp. 1464-1467.

${ }^{7}$ O. Berger, D. Inns, and A. G. Aberle, Sol. Energy Mater. Sol. Cells 91, 1215 (2007).

${ }^{8}$ S. Faÿ, J. Steinhauser, N. Oliveira, E. Vallat-Sauvain, and C. Ballif, Thin Solid Films 515, 8558 (2007).

${ }^{9}$ A. Hedler, J. Eberhardt, C. Wachtendorf, S. Correia, R. Gutlapalli, D. Messerschmidt, and C. Koitzsch, Proceedings of the 34th IEEE Photovoltaic Specialists Conference, Philadelphia, Pennsylvania, 7-12 June 2009, pp. 1102-1106.

${ }^{10}$ J. E. Cotter, J. Appl. Phys. 84, 618 (1998).

${ }^{11}$ W. E. Vargas, P. Greenwood, J. E. Otterstedt, and G. A. Niklasson, Sol. Energy 68, 553 (2000)

${ }^{12}$ W. E. Vargas, A. Amador, and G. A. Niklasson, Opt. Commun. 261, 71 (2006).

${ }^{13}$ P. Nitz, J. Ferber, R. Stangl, H. R. Wilson, and V. Wittwer, Sol. Energy Mater. Sol. Cells 54, 297 (1998).

${ }^{14}$ J. Springer, A. Poruba, and M. Vanecek, J. Appl. Phys. 96, 5329 (2004).

${ }^{15}$ B. E. Pieters, J. Krč, and M. Zeman, Proceedings of the 4th IEEE World Conference on Photovoltaic Energy Conversion, Waikoloa, Hawaii, 8-12 May 2006, pp. 1513-1516.

${ }^{16}$ J. Krč, F. Smole, and M. Topič, Prog. Photovoltaics 11, 15 (2003).

${ }^{17}$ A. Goldschmidt and H. Streitberger, Basics of Coating Technology (Vincentz Network, Hannover, Germany, 2003).

${ }^{18}$ J. E. Cotter, R. B. Hall, M. G. Mauk, and A. M. Barnett, Prog. Photovoltaics 7, 261 (1999).

${ }^{19}$ M. J. Webber, Handbook of optical materials (CRC, Boca Raton, FL, 2003)

${ }^{20}$ M. R. Shenoy, IEE Proc.-J: Optoelectron. 139, 163 (1992).

${ }^{21}$ G. P. Smestad, Optoelectronics of Solar Cells (SPIE, Bellingham, Washington, 2002)

${ }^{22}$ M. Born and E. Wolf, Principles of Optics (Cambridge University Press, Cambridge, 1999).

${ }^{23} \mathrm{P}$. Beckmann and A. Spizzichino, The Scattering of Electro-Magnetic Waves from Rough Surfaces (Pergamon, New York, 1963).

${ }^{24}$ C. K. Carniglia, Opt. Eng. 18, 104 (1979).

${ }^{25}$ J. Krč, M. Zeman, F. Smole, and M. Topič, J. Appl. Phys. 92, 749 (2002).

${ }^{26}$ M. Zeman, R. A. C. M. M. Van Swaaij, and J. W. Metselaar, J. Appl. Phys. 88, 6436 (2000).

${ }^{27}$ F. C. Krebs, Sol. Energy Mater. Sol. Cells 93, 394 (2009). 